Conclusion: The results call for a comprehensive understanding of the scene: the teams are working in a scene that has not been secured, with possible presence of additional perpetrators. Personnel has to work using Personal Protective Equipment (PPE) due to that risk. Dealing with an injured perpetrator requires security checks, authorization of the security authorities on the scene, and moral dilemmas. Transportation times might be prolonged. This creates a unique environment that calls for specific on-scene protocols, as well as training of the personnel (staff and volunteers) to be able to successfully perform their tasks in this hostile environment. On-scene procedures, as well as unique procedures developed (eg, police escort to overcome traffic), and revised treatment protocols as result of lessons learned from incidents will be presented.

Prehosp Disaster Med 2017;32(Suppl. 1):s118-s119

doi:10.1017/S1049023X17003375

Clinical Care for Sexual Assault Survivors (CCSAS): the Use of a Multimedia Training Tool. Nagi Souaiby

Faculty of Medicine, St Joseph University, Beirut/Lebanon

Study/Objective: Evaluate a multimedia training tool used to train Health Care Providers (HCPs) as key actors in improving the delivery of quality Clinical Care for Sexual Assault Survivors (CCSAS).

Background: Sexual assault rises as a global public health issue, in conflict-affected populations, where SGBV becomes a strategy of war. Training HCPs has been prioritized by humanitarian actors globally to improve the quality CCSAS. Few studies have evaluated the effectiveness of such training.

Methods: Four ToTs days were provided to relevant community HCPs working in a conflict area in Jordan, Turkey, Syria, and Lebanon. The CCSAS multi-media tool developed by the IRC was used as a unified training tool aiming to improve clinical care. The recruitment process included a general call for application, entailing a detailed syllabus for the training course whereby individuals expressed their interest in attending and submitted their resume to ensure that their qualifications were in-line with the pre-set selection criteria for the training.

Results: Six ToTs took place; in Jordan, two groups of 25 have improved by $142 \%$ and $57.6 \%$ on average at post-test in knowledge and attitudes to care for survivors. The third ToT in Turkey, 13 participants have improved by $47 \%$ on average and nine participants have improved by $82.6 \%$ on average. In Lebanon, 19 participants have improved by $62.5 \%$ on average. In Syria, 18 participants have improved by $46.2 \%$ on average. Key barriers to quality care identified included poor or lack of access to services, lack of privacy and confidentiality, and lack of essential resources and treatment including PEP, as well as an unclear referral mechanism. Action plans were developed by participants to address these barriers and follow-up to the evaluating progress was planned.

Conclusion: The CCSAS multi-media training tool showed an initial positive impact and has demonstrated effectiveness in promoting compassion and competence among trained HCPs and improving quality of care in humanitarian settings.

Prehosp Disaster Med 2017;32(Suppl. 1):s119

doi:10.1017/S1049023X17003387

Paris Terrorist Attack on November 13, 2015 - Applying Wartime In-hospital Triage and Damage Control Strategies Bertrand Grand ${ }^{1}$, Guillaume Boddaert ${ }^{1}$, Jean Louis Daban ${ }^{2}$, Emmanuel Hornez ${ }^{3}$, Anne De Carbonnieres ${ }^{3}$, Guillaume Giral ${ }^{3}$, Dary Ngabou ${ }^{1}$,Amélie Mlynski ${ }^{1}$, Federico Gonzalez ${ }^{3}$, Tarun Mcbride ${ }^{1}$, Stéphane Bonnet ${ }^{3}$

1. Thoracic And Vascular Surgery, Percy Military Teaching Hospital, Clamart/France

2. Department Of Anesthesia And Intensive Care,Percy Military Teaching Hospital, CLAMART/France

3. Digestive And General Surgery Department, Percy Military Teaching Hospital, CLAMART/France

Study/Objective: The Paris terrorist attack of November $13^{\text {th }}$ 2015 caused 130 deaths and 351 injured.

Background: Our work aims to show how in-hospital triage and damage control strategies, acquired during the recent conflicts in Afghanistan and Sahel, enable a rational and appropriate management of the patients.

Methods: We retrospectively reviewed the cohort of 17 patients treated at the Percy Military Teaching Hospital on the nights of November 13-14, 2015.

Results: The mean age was $39 \pm 8$ years. Eight patients (47\%) had a thoracic injury (mean AIS $=3[1-6]), 5(29 \%)$ an upper limb injury (mean AIS = 2[1-3]), 4 (24\%) an abdominal injury (mean AIS $=3[2-4]), 3(18 \%)$ a face injury (AIS $2=$ medium [1-3]), $3(18 \%)$ a lower limb injury (AIS = 1), $2(12 \%)$ a spine injury (AIS $=5)$ and $1(6 \%)$ a brain injury (AIS $=5)$. There was no patient identity error. Two patients (12\%) were categorized immediate with extreme mention (T1E) (ISS 19 and 29), 6 (35\%) immediate (T1) (average ISS = 24 [13-41]), $4(24 \%)$ delayed (T2) (average ISS $=6$ [1-16]) and $5(29 \%)$ minimal (T3) (average ISS = 1 [1-3]). Four patients $(24 \%)$ had a damage control procedure with a mean surgical time of 68 min (43-84). All patients were treated according to the deadlines imposed by their categorization. One patient died of multiple organ failure in the aftermath of a resuscitation thoracotomy. All patient records were reviewed and three were analyzed as perfectible, without consequences for the patients involved.

Conclusion: The current context exposes us to the threat of new possible terrorist attacks and requires that the medical community get prepared to manage multiple war casualties. The familiarization to the modern principles of war surgery seems mandatory to face this type of situation.

Prehosp Disaster Med 2017;32(Suppl. 1):s119

doi:10.1017/S1049023X17003399

Lessons of Military Anesthesiologists after Terror Attacks in Paris. Comparison with Battlefield Experience. Jean Louis Daban ${ }^{1}$, Nicolas Donat ${ }^{2}$, Philippe Laitselart ${ }^{1}$, Guillaume Boddaert ${ }^{3}$, Emmanuel Hornez ${ }^{4}$, Guillaume De Saint Maurice $^{1}$ 
1. Department Of Anesthesia And Intensive Care, Percy Military Teaching Hospital, CLAMART/France

2. Burn Center, Percy Military Teaching Hospital, CLAMART/ France

3. Department Thoracic Surgery Department, Percy Military Teaching Hospital, CLAMART/France

4. Digestive And General Surgery Department, Percy Military Teaching Hospital, CLAMART/France

Study/Objective: Objective of this study is to report the management by a military hospital of an influx of civilian casualties due to terror attacks.

Background: During the night of November 13, 2015, Percy army teaching hospital received 17 wounded in two convoys.

Methods: Retrospective, descriptive study

Results: Except one, all patients (8 AU and 9 RU) had GSW and nearly half of the patients had thoracic injuries (no body armor) in contrast to recent military series. Triage of GSW was easier than injuries by explosion. The hospital was away from sites of the attacks with a delay of two hours before the arrival of the first patient. Five trauma bays for AU were prepared with for each 1 anesthesiologist, 1 nurse, 1 anesthetist nurse. Eight patients were hospitalized in ICU. A binomial surgeon-anesthetist was in charge of triage which is the rule in French army. Before arrival of first casualties, bracelets with a temporary identity were generated to avoid any mistake of identity. During the first hours; 32 PRBC, 32 lyophilized plasmas and 3 platelet concentrates were transfused without incident. The use of the universal lyophilized plasma specific to the French army facilitated immediate transfusion. Prior to the arrival of the first patient, it was decided that only damage control procedures would be authorized regardless of the clinical condition (collective indications of damage control). The goal of this decision was to optimize the use of operating theaters. This decision was motivated by the risk of second attacks and experience of managing a large number of wounded with a limited operating theaters number during deployments. Operating theaters like the rest of the hospital were never saturated. As in times of war, the transmission of information between the different levels of care was difficult.

Conclusion: Military skills are useful in managing an influx of casualties.

Prehosp Disaster Med 2017;32(Suppl. 1):s119-s120

doi:10.1017/S1049023X17003405

Pray for the Best, Prepare for the Worst: Cholera Treatment Ward Preparation of Novice Haitian Healthcare Staff Sean Smith

Critical-care Professionals International, Critical-Care Professionals International, Durham/United States of America

Study/Objective: To describe the preparing of Haitian Healthcare staff for triaging and management of cholera patients.

Background: Haiti has endemic cholera. This is exacerbated by our (yearly) rainy season. In 2016, this was further worsened by Hurricane Matthew, allowing massive contamination of water supplies in southern Haiti. This resulted in a much greater need for Haitian healthcare workers with no previous exposure to this patient population, to be able to effectively recognize, treat and contain potential cholera patients.

Methods: Observational discussion and lessons learned from setting up a Cholera Treatment Ward in Southern Haiti, post Hurricane Matthew.

Results: Haitian Healthcare workers with no prior experience or capacity for treating cholera developed an effective knowledge base, skillset, and Cholera Treatment Ward (CTW), thereby proactively heightening local disaster management capabilities.

Conclusion: Many of our lessons learned are applicable to a wide variety of disasters, infectious diseases, capacity building situations and would be of interest to WADEM members' attendees.

Prehosp Disaster Med 2017;32(Suppl. 1):s120

doi:10.1017/S1049023X17003417

\section{Does Planning and Preparation Help in Disaster Risk Management? A Nepal Experience \\ Pradeep Vaidya}

Department of Surgery, Tribhuvan University Teaching Hospital, Kathmandu/Nepal

Study/Objective: To review the response mechanism and the preparation before the Nepal earthquake, for effective preparation and response.

Background: Nepal which lies between two large countries of China and India and is prone to natural disasters including earthquakes, floods, landslides and severe weather events. Bureau for Crisis Prevention and Recovery (BCPR) ranks Nepal as the 11th most at risk country in the world in terms of relative vulnerability to earthquake. The (last year) earthquake in Nepal had caused nearly 9,000 deaths compared to the expected 100,000 deaths, and this is due to the timing of the event. The earthquake occurred on Saturday, so all the schools and offices were on holiday, that is the one important reason for the lower number of casualties; and the other is the preparedness of the Ministry of Health and the hospitals. The first factor was not in our hands to modify but the second one was. Two factors, one was that the government had taken the lead of all the disaster preparedness by various International NonGovernmental Organizations (INGOs) through the cluster system. The Second is the training in disaster preparedness by the course called Hospital Preparedness for Emergencies developed by US Aid.

Methods: Hospital Preparedness for Emergencies (HOPE), Primary Trauma Care (PTC), Emergency protocols were conducted and the Health Emergency Operation Center (HEOC) was prepared before the earthquake which helped in saving many lives. The HUB hospital system was implemented by Ministry of Health to coordinate better among the hospitals. Results: All of this preparedness may not have worked completely, but the review organized by WHO and Ministry of Health, Nepal after the disaster showed that these training protocols and HEOC had really helped to treat patients systematically. 